\title{
Reliability and Profit Analysis of a Two-unit Non-identical Standby System in Snowstorm Weather Conditions
}

\author{
Narender Singh", Dalip Singh, Meenaxi \\ Department of mathematics, M.D. University, Rohtak, India \\ *Corresponding author: shiviswarhansunandu.1988@email
}

\begin{abstract}
This paper express reliability measures of a cold standby system which have two units. In cold standby system one unit operative and other unit kept as a spare. In the system both the unit kept as non-identical. The each operative unit fails due to snowstorm with different failure rate. The system completely failed when the both two units are failed. The failed unit cannot be operative directly by the repairman. The failed unit under the snow, first digging out from the snow then hospitalize (repair) the unit after that the unit becomes operative. Some properties of reliability system such as mean time to system failure, availability and profit have been computed. At last particular cases have been taken to explain the model.
\end{abstract}

Keywords: non-identical units, snowstorms, digging out

Cite This Article: Narender Singh, Dalip Singh, and Meenaxi, "Reliability and Profit Analysis of a Two-unit Non-identical Standby System in Snowstorm Weather Conditions." American Journal of Applied Mathematics and Statistics, vol. 6, no. 3 (2018): 109-114. doi: 10.12691/ajams-6-3-4.

\section{Introduction}

Today in modern industry, reliability has an important role for the system. Reliability models of two-unit standby systems have been analysed by various research including [1,2,3]. Taneja and Tuteja [4,5] discussed various systems with different types of failure and repair rates. Comparative analysis of two-unit standby systems are studied by Singh and Taneja [6] and Malhotra and Taneja [7]. Chandrasekhar P. et al. [8] focused on two unit system with erlangian repair time. Manocha and Taneja [9] worked on such systems with arbitrary distributions. Reliability of a system most affected by abnormal weather conditions such as heavy rain snowstorm, dense fog, high temperature etc. Therefore, many researchers including Goel, Sharma and Gupta [10], Gupta and Goel [11] and Goel, Kumar and Rastogi [12] have explained reliability measures of systems with various weather conditions. Singh et al. [13] analyzed availability of warm standby systems failure due to heavy rain. Nailwal and Singh [14] analysed reliability and sensitivity in different weather conditions.

In this paper, we consider the two-unit non-identical units system. The system has only one repairman as a rescue team. The operative unit failed due to snow storm and some people or systems were trapped under the snow. In such situation repair of the system is very difficult. So after the snowstorm is over, first the failed unit digging out by the repairman. after the digging out then hospitalize the such unit and after that the unit becomes operative.
This paper describes the following subsections and sections.

- Model with mean sojourn times and transition probabilities

- Mean time to system failure

- Steady state availability

- Repairman's busy period analysis during digging out

- Repairman’s busy period analysis during hospitalization

- Repairman's expected visits

- Cost-Benefit analysis

- Special case

- Graphical Explanation

\section{Notations}

$\lambda_{1}$ : failure rate of first unit due to snow storm

$\lambda_{2}$ : failure rate of second unit due to snow storm

$G_{1}(t), G_{2}(t)$ : comulative density function of first unit as repair rate of digging out and hospitilization of failed unit respectively.

$G_{3}(t), G_{4}(t)$ : comulative density function of second unit as repair rate of digging out and hospitilization of failed unit respectively.

$\mathrm{g}_{1}(\mathrm{t}), \mathrm{g}_{2}(\mathrm{t})$ : probability density function of first unit as repair rate of digging out, and hospitilization of failed unit respectively.

$\mathrm{g}_{3}(\mathrm{t}) \mathrm{g}_{4}(\mathrm{t})$ : probability density function of second unit as the repair rate of digging out and hospitilization of failed unit respectively.

Op: operative unit 
cs: spare unit or cold standby unit

Fd: failed unit is under digging out

FD: failed unit is under digging out continuing on the unit

Fh: failed unit is under hospitalization after snow removing

FH: failed unit is under hospitalization continuing after snow removing

Fwd: waiting for digging out

\subsection{Model and Transition Probability:}

In the state transition diagram (Figure 1) states 0, 1, 2, 4, $5,8,9$ are regenerative states and 3,6,7,10 and nonregenerative states.

$p_{01}=1$,

$p_{12}=g_{1}^{*}\left(\lambda_{2}\right), p_{13}=\left(1-g_{1}^{*}\left(\lambda_{2}\right)\right), p_{14}^{3}=\left(1-g_{1}^{*}\left(\lambda_{2}\right)\right)$

$p_{20}=g_{2}^{*}\left(\lambda_{2}\right), p_{26}=\left(1-g_{2}^{*}\left(\lambda_{2}\right)\right), p_{25}^{6}=\left(1-g_{2}^{*}\left(\lambda_{2}\right)\right)$

$\mathrm{p}_{45}=1, p_{90}=g_{4}^{*}\left(\lambda_{1}\right), p_{91}^{10}=\left(1-g_{4}^{*}\left(\lambda_{1}\right)\right)$

$\left.p_{81}=1, p_{59}=g_{3}^{*}\left(\lambda_{1}\right)\right), p_{58}^{7}=\left(1-g_{3}^{*}\left(\lambda_{1}\right)\right)$.
By these transition probabilities, it can be verified that $p_{01}=1$

$p_{12}+p_{14}^{(3)}=p_{12}+p_{13}=1=p_{59}+p_{58}^{(7)}$

$p_{20}+p_{25}^{(6)}=p_{20}+p_{26}=1=p_{90}+p_{91}^{(10)}$ $p_{45}=1=p_{81}$.

If $T$ represents the sojourn then mean sojourn time $\left(\mu_{\mathrm{i}}\right)$ at the regenerative state ' $\mathrm{i}$ ' discussed as:

$$
\begin{aligned}
& \mu_{\mathrm{i}}=\mathrm{E}(\mathrm{T})=\operatorname{Pr}(\mathrm{T}>\mathrm{t}) \\
& \mu_{0}=\frac{1}{\lambda_{1}} \\
& \mu_{1}=\frac{1}{\lambda_{2}}\left\{1-g_{1}^{*}\left(\lambda_{2}\right)\right\} \\
& \mu_{2}=\frac{1}{\lambda_{2}}\left\{1-g_{2}^{*}\left(\lambda_{2}\right)\right\} \\
& \mu_{5}=\frac{1}{\lambda_{1}}\left\{1-g_{3}^{*}\left(\lambda_{2}\right)\right\} \\
& \mu_{9}=\frac{1}{\lambda_{1}}\left\{1-g_{4}^{*}\left(\lambda_{2}\right)\right\} .
\end{aligned}
$$

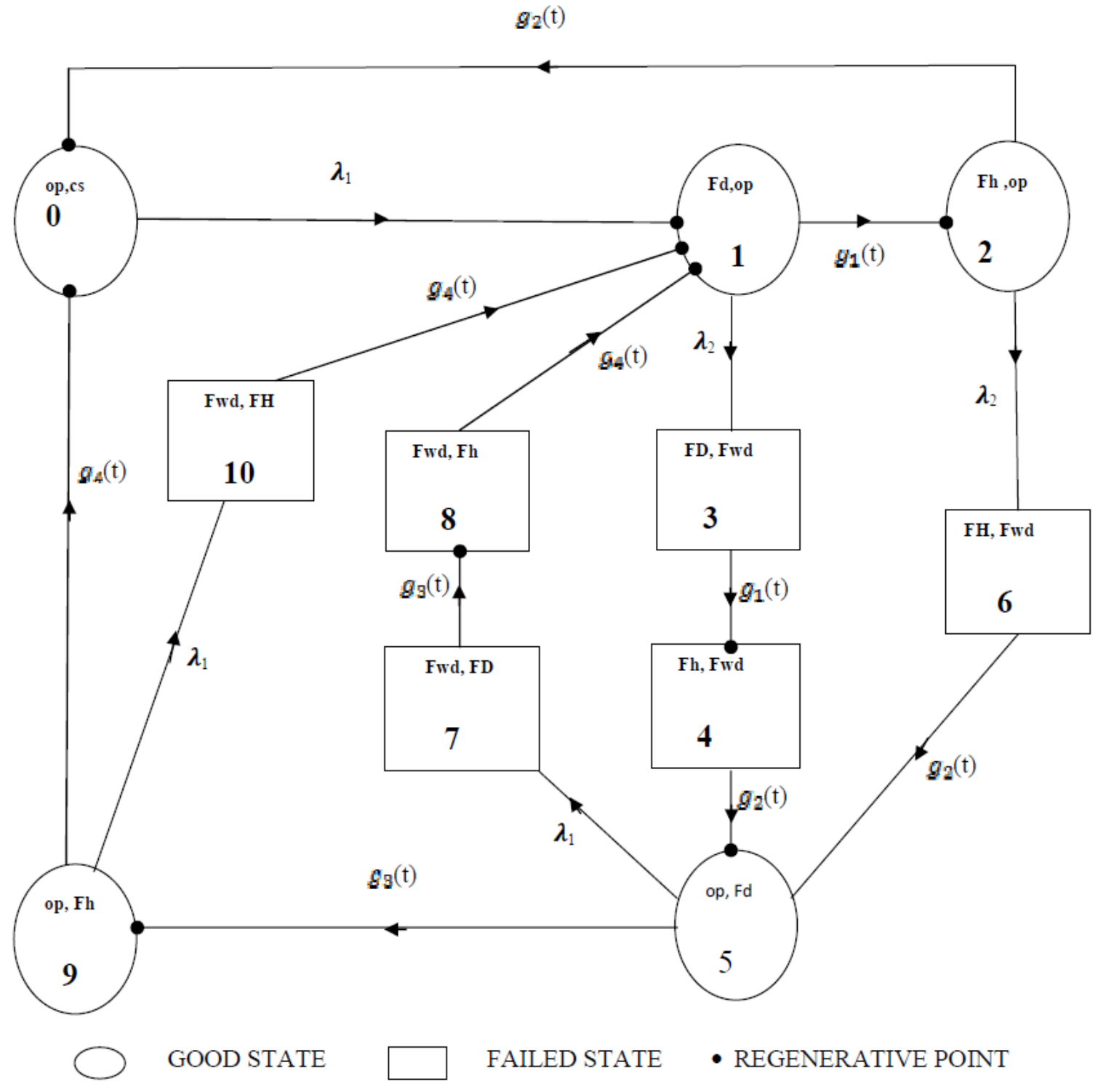

Figure 1. 
The unconditional mean time $\mathrm{m}_{\mathrm{ij}}$ mathematically defined as

$$
\begin{gathered}
m_{i j}=\int_{0}^{\infty} t q_{i j}(\mathrm{t}) \mathrm{dt}=-q_{i j}^{*^{\prime}}(0) \\
m_{01}=\mu_{0} \\
m_{12}+m_{14}^{3}=-g_{1}^{*^{\prime}}(0)=k_{1} \text { (say), } m_{12}+m_{14}=\mu_{1} \\
m_{20}+m_{25}^{6}=-g_{2}^{*^{\prime}}(0)=k_{2}(\text { say }), m_{20}+m_{26}=\mu_{2} \\
m_{45}=k_{2}, m_{59}+m_{58}^{7}=-g_{3}^{*^{\prime}}(0)=k_{3} \text { (say), } \\
m_{81}=k_{4}, m_{90}+m_{91}^{10}=-g_{4}^{*^{\prime}}(0)=k_{4} \text { (say). }
\end{gathered}
$$

\section{Mean Time to System Failure}

Mean time to system failure (MTSF) regarding the failed states (i=3, 4, 6, 7, 8, 10) as absorbing states and applying arguments for regenerative process, we get the recursion relation for $\pi_{i}(t)$,

$$
\begin{gathered}
\pi_{0}(t)=Q_{01}(t) \text { (S) } \pi_{1}(t), \\
\pi_{1}(t)=Q_{13}(t)+Q_{12}(t) \text { (S) } \pi_{2}(t) \\
\pi_{2}(t)=Q_{26}(t)+Q_{20}(t) \text { (S) } \pi_{0}(t) .
\end{gathered}
$$

By applying Laplace-Stieltjes transform on these relations and solving for $\pi_{i}^{* *}(\mathrm{~s})=\frac{N(S)}{D(S)}$.

Where, $\mathrm{N}(\mathrm{S})=Q_{01}^{* *}(\mathrm{~s})\left(Q_{13}^{* *}(\mathrm{~s})+Q_{26}^{* *}(\mathrm{~s}) Q_{12}^{* *}(\mathrm{~s})\right)$

$\mathrm{D}(\mathrm{S})=1-Q_{01}^{* *}(\mathrm{~s}) Q_{20}^{* *}(\mathrm{~s}) Q_{12}^{* *}(\mathrm{~s})$.

When the system begin from the state ' 0 ' the mean time to system failure is

$$
\begin{aligned}
& T_{0}=\lim _{s \rightarrow 0} \frac{1-\phi_{0}^{* *}(s)}{s}=\lim _{s \rightarrow 0} \frac{1-\frac{N(S)}{D(S)}}{s} \\
& =\lim _{s \rightarrow 0} \frac{D(s)-N(s)}{s D(s)}=\frac{D^{\prime}(0)-N^{\prime}(0)}{D(0)}=\frac{N}{D}
\end{aligned}
$$

Where $\mathrm{N}=\mu_{0}+p_{12} \mu_{2}+\mu_{1}$

And

$$
\mathrm{D}=1-p_{12} p_{20}
$$

\section{Availability Analysis}

System availability is the probability that it is in operation and gives service when we want.

By using the theory of regenerative point process, availability $\mathrm{A}_{\mathrm{i}}(\mathrm{t})$ as the probability in the state ' $\mathrm{i}$ ' at $\mathrm{t}=0$ is seen to satisfy these recursive relation are obtained.

$$
\begin{aligned}
& A_{0}(t)=M_{0}(\mathrm{t})+q_{01}(\mathrm{t})\left(A_{1}(\mathrm{t})\right. \\
& A_{1}(\mathrm{t})=M_{1}(\mathrm{t})+q_{12}(\mathrm{t})\left(A_{2}(\mathrm{t})+q_{15}^{4}(\mathrm{t})\left(A_{5}(\mathrm{t})\right.\right. \\
& A_{2}(\mathrm{t})=M_{2}(\mathrm{t})+q_{20}(\mathrm{t})\left(A_{0}(\mathrm{t})+q_{25}^{6}(\mathrm{t})\left(A_{5}(\mathrm{t})\right.\right. \\
& A_{4}(t)=q_{45}(\mathrm{t}) \cong A_{5}(\mathrm{t}) \\
& A_{5}(t)=M_{5}(\mathrm{t})+q_{59}(\mathrm{t})\left(A_{9}(\mathrm{t})+q_{58}^{7}(\mathrm{t})\left(\mathrm{C} A_{8}(\mathrm{t})\right.\right. \\
& A_{8}(t)=q_{81}(\mathrm{t})\left(A_{1}(\mathrm{t})\right. \\
& A_{9}(t)=M_{9}(\mathrm{t})+q_{90}(\mathrm{t})\left(A_{0}(\mathrm{t})+q_{91}^{10}(\mathrm{t})\left(A_{1}(\mathrm{t})\right.\right.
\end{aligned}
$$

Where $M_{0}(\mathrm{t})=e^{-\lambda_{1} t} d t, M_{1}(\mathrm{t})=e^{-\lambda_{2} t} \overline{G_{1}}(\mathrm{t}) \mathrm{dt}, M_{2}(\mathrm{t})=$ $e^{-\lambda_{2} t} \overline{G_{2}}(\mathrm{t}) \mathrm{dt}, M_{5}(\mathrm{t})=e^{-\lambda_{1} t} \overline{G_{3}}(\mathrm{t}) \mathrm{dt}$ and $M_{9}(\mathrm{t})=e^{-\lambda_{1} t} \overline{G_{4}}(\mathrm{t}) \mathrm{dt}$.

By applying Laplace-Transformations on these relation and solving for $A_{0}^{*}(s)$ we get,

$$
A_{0}^{*}(\mathrm{~s})=\frac{N_{1}(s)}{D_{1}(\mathrm{~s})}
$$

availability of system in steady state is

$$
A_{0}=\lim _{s \rightarrow 0}\left(s A_{0}^{*}(s)\right)=\lim _{s \rightarrow 0}\left(s \frac{N_{1}(s)}{D_{1}(s)}\right)=\frac{N_{1}(0)}{D_{1}^{\prime}(0)}=\frac{N_{1}}{D_{1}}
$$

$$
\begin{aligned}
N_{1}= & \mu_{0}\left(1-p_{12} p_{25}^{6} p_{91}^{10}-p_{12} p_{25}^{6} p_{58}^{7}-p_{14}^{3} p_{91}^{10}-p_{14}^{3} p_{58}^{7}\right) \\
& +\mu_{1}+\mu_{2} p_{12}+\left(\mu_{5}+\mu_{5}\right)\left(p_{12} p_{25}^{6}+p_{14}^{3}\right) \\
D_{1}= & k_{1}+k_{2}+\mu_{0}\left(p_{12} p_{20}+p_{12} p_{25}^{6} p_{59} p_{90}+p_{14}^{3} p_{59} p_{90}\right) \\
& +\left(k_{3}+k_{4}\right)\left(p_{12} p_{25}^{6}+p_{14}^{3}\right)
\end{aligned}
$$

Where, $k_{1}, k_{2}, k_{3}$ and $k_{4}$ is already defined.

\section{During Digging out Repairman Busy Period Analysis}

$B_{i}^{D}(\mathrm{t})=$ The system entered from regenerative state ' $\mathrm{i}$ 'at time $\mathrm{t}=0$ is under repair during digging out.

$$
\begin{aligned}
& B_{0}^{D}(t)=q_{01}(\mathrm{t})(C) B_{1}^{D}(\mathrm{t}) \\
& B_{1}^{D}(t)=W_{1}(\mathrm{t})+q_{12}(\mathrm{t}) \subset B_{2}^{D}(\mathrm{t})+q_{15}^{4}(\mathrm{t})\left(B_{4}^{D}(\mathrm{t})\right. \\
& B_{2}^{D}(t)=q_{20}(\mathrm{t}) \subseteq B_{0}^{D}(\mathrm{t})+q_{25}^{6}(\mathrm{t})\left(B_{5}^{D}(\mathrm{t})\right. \\
& B_{4}^{D}(t)=q_{45}(\mathrm{t})(C) B_{5}^{D}(\mathrm{t}) \\
& B_{5}^{D}(t)=W_{5}(\mathrm{t})+q_{59}(\mathrm{t}) \subset B_{9}^{D}(\mathrm{t})+q_{58}^{7}(\mathrm{t}) \subset B_{8}^{D}(\mathrm{t}) \\
& B_{8}^{D}(t)=q_{81}(\mathrm{t})\left(B_{1}^{D}(\mathrm{t})\right. \\
& B_{9}^{D}(t)=W_{9}(\mathrm{t})+q_{90}(\mathrm{t}) \subset B_{0}^{D}(\mathrm{t})+q_{91}^{10}(\mathrm{t}) \subset B_{1}^{D}(\mathrm{t})
\end{aligned}
$$

Where $W_{2}(\mathrm{t})=e^{-\lambda_{2} t} \overline{G_{2}}(\mathrm{t}) \mathrm{dt}+\lambda_{2} e^{-\lambda_{2} t} \overline{G_{2}}(\mathrm{t}) \mathrm{dt}, W_{9}(\mathrm{t})=$ $e^{-\lambda_{1} t} \overline{G_{4}}(\mathrm{t}) \mathrm{dt}+\lambda_{1} e^{-\lambda_{1} t} \overline{G_{9}}(\mathrm{t}) \mathrm{dt}, W_{8}(\mathrm{t})=\overline{G_{3}}(\mathrm{t}) \mathrm{dt}$ and $W_{4}(\mathrm{t})=\overline{G_{2}}(\mathrm{t}) \mathrm{dt}$.

By using Laplace Transforms then solving system of equation for $B_{0}^{* H}(s)$ we get,

$$
B_{0}^{* H}(s)=\frac{N_{3}(s)}{D_{1}(s)}
$$

Where

$$
\begin{aligned}
N_{3}(s)= & q_{01}^{*}(s) q_{14}^{*(3)}(s) W_{4}^{*}(s) \\
& +q_{01}^{*}(s) q_{12}^{*}(\mathrm{~s}) W_{2}^{*}(\mathrm{~s})+q_{01}^{*}(s) q_{14}^{*(3)}(s) \\
& +q_{45}^{*}(s) q_{58}^{*(7)}(s) W_{8}^{*}(s)+q_{01}^{*}(s) q_{14}^{*(3)}(s) \\
& +q_{45}^{*}(s) q_{59}^{*}(\mathrm{~s}) W_{9}^{*}(s) \\
& +q_{01}^{*}(\mathrm{~s}) q_{12}^{*}(\mathrm{~s}) q_{25}^{*(6)}(\mathrm{s}) q_{58}^{*(7)}(s) W_{8}^{*}(s) \\
& +q_{01}^{*}(\mathrm{~s}) q_{12}^{*}(\mathrm{~s}) q_{25}^{*(6)}(\mathrm{s}) q_{59}^{*}(\mathrm{~s}) W_{9}^{*}(s) .
\end{aligned}
$$

where $D_{1}(\mathrm{~s})$ defined already. 
During digging out the total time for which the system is under repaired.

$$
B_{0}^{* H}=\frac{N_{3}(0)}{D_{1}^{\prime}(0)}=\frac{N_{3}}{D_{1}}
$$

Where,

$$
N_{2}=W_{1}+\left(p_{12} p_{25}^{6}+p_{14}^{3} p_{45}\right) W_{5}
$$

Where $W_{1}=W_{1}^{*}(0)$ and $D_{1}$ is already defined.

\section{During Hospitalization Repairman Busy Period Analysis}

$B_{i}^{H}(\mathrm{t})=$ The system entered from regenerative state ' $\mathrm{i}$ 'at time $\mathrm{t}=0$ is under repair during digging out.

$$
\begin{aligned}
& B_{0}^{H}(t)=q_{01}(\mathrm{t}) \text { C } B_{1}^{H}(\mathrm{t}) \\
& B_{1}^{H}(t)=q_{12}(\mathrm{t}) \subset B_{2}^{H}(\mathrm{t})+q_{15}^{4}(\mathrm{t}) \subset B_{4}^{H}(\mathrm{t}) \\
& B_{2}^{H}(t)=W_{2}(\mathrm{t})+q_{20}(\mathrm{t}) \subset B_{0}^{H}(\mathrm{t})+q_{25}^{6}(\mathrm{t}) \subset B_{5}^{H}(\mathrm{t}) \\
& B_{4}^{H}(t)=W_{4}(\mathrm{t})+q_{45}(\mathrm{t}) \subset B_{5}^{H}(\mathrm{t}) \\
& B_{5}^{H}(t)=q_{59}(\mathrm{t}) \subset B_{9}^{H}(\mathrm{t})+q_{58}^{7}(\mathrm{t}) \subset B_{8}^{H}(\mathrm{t}) \\
& B_{8}^{H}(t)=W_{8}(\mathrm{t})+q_{81}(\mathrm{t}) \text { C } B_{1}^{H}(\mathrm{t}) \\
& B_{9}^{H}(t)=W_{9}(\mathrm{t})+M_{9}(\mathrm{t})+q_{90} \subset B_{0}^{H}(\mathrm{t})+q_{91}^{10}(\mathrm{t}) \subset B_{1}^{H}(\mathrm{t})
\end{aligned}
$$

Where $W_{1}(\mathrm{t})=e^{-\lambda_{2} t} \overline{G_{1}}(\mathrm{t}) \mathrm{dt}+\lambda_{2} e^{-\lambda_{2} t} \overline{G_{2}}(\mathrm{t}) \mathrm{dt}, W_{5}(\mathrm{t})=$ $e^{-\lambda_{1} t} \overline{G_{3}}(\mathrm{t}) \mathrm{dt}+\lambda_{1} e^{-\lambda_{1} t} \overline{G_{3}}(\mathrm{t}) \mathrm{dt}$

By using Laplace Transforms then solving system of equation for $B_{0}^{* D}(s)$ we get,

$$
B_{0}^{* D}(s)=\frac{N_{2}(s)}{D_{1}(s)}
$$

Where

$$
N_{2}(s)=q_{01}^{*}(s) W_{1}^{*}(s)+\left(\begin{array}{c}
q_{01}^{*}(s) q_{12}^{*}(\mathrm{~s}) q_{25}^{*(6)}(s) \\
+q_{01}^{*}(s) q_{14}^{*(3)}(\mathrm{s}) q_{45}^{*}(s)
\end{array}\right) W_{5}^{*}(s)
$$

where $D_{1}(\mathrm{~s})$ is already defined.

During hospitalization the total time for which the system is under repaired.

$$
B_{0}^{* H}=\frac{N_{3}(0)}{D_{1}^{\prime}(0)}=\frac{N_{3}}{D_{1}}
$$

Where,

$$
\begin{aligned}
N_{3}= & W_{4} p_{14}^{(3)}+p_{12} W_{2}(t)+p_{14}^{(3)} p_{58}^{(7)} W_{8}+p_{14}^{(3)} p_{59} W_{9} \\
& +p_{12} p_{25}^{(6)} p_{58}^{(7)} W_{8}+p_{12} p_{25}^{(6)} p_{59} W_{9}
\end{aligned}
$$

Where $W_{2}=W_{2}^{*}(0), W_{4}=W_{4}^{*}(0), W_{8}=W_{8}^{*}(0), W_{9}=W_{9}^{*}(0)$ and $D_{1}$ is defined already.

\section{Expected Number of Visits by the Repairman}

When the system started from the regenerative state ' $\mathrm{i}$ ' at $\mathrm{t}=0, V_{0}(\mathrm{t})$ denotes the expected number of visits by the repair mean in $[0, \mathrm{t}]$.

$$
\begin{aligned}
& V_{0}(t)=q_{01}(\mathrm{t}) \mathrm{S}\left(1+V_{1}(\mathrm{t})\right) \\
& V_{1}(t)=q_{12}(\mathrm{t}) \mathrm{S} V_{2}(\mathrm{t})+q_{15}^{4}(\mathrm{t})(\mathrm{S}) V_{5}(\mathrm{t}) \\
& V_{2}(t)=q_{20}(\mathrm{t})(\mathrm{S}) V_{0}(\mathrm{t})+q_{25}^{6}(\mathrm{t}) \subseteq V_{5}(\mathrm{t}) \\
& V_{4}(t)=q_{45}(\mathrm{t}) \mathrm{S} V_{5}(\mathrm{t}) \\
& V_{5}(t)=q_{59}(\mathrm{t}) \subseteq V_{9}(\mathrm{t})+q_{58}^{7}(\mathrm{t})(\mathrm{S}) V_{8}(\mathrm{t}) \\
& V_{8}(t)=q_{81}(\mathrm{t})(\mathrm{S}) V_{1}(\mathrm{t}) \\
& V_{9}(t)=q_{90}(\mathrm{t}) \subseteq V_{0}(\mathrm{t})+q_{91}^{10}(\mathrm{t}) \subseteq V_{1}(\mathrm{t}) \text {. }
\end{aligned}
$$

By using Laplace-Stieltjes Transformations and solving system of equations for $V_{0}^{* *}(s)$, we get

$$
V_{0}^{* *}(s)=\frac{N_{3}(s)}{D_{1}(s)}
$$

Where

$$
\begin{aligned}
& N_{4}(s)=Q_{01}^{* *}(s)-Q_{12}^{* *}(\mathrm{~s}) Q_{25}^{* *(6)}(s) Q_{59}^{* *}(\mathrm{~s}) Q_{91}^{* *(10)} Q_{01}^{* *}(\mathrm{~s})[ \\
& -Q_{12}^{* *}(\mathrm{~s}) Q_{25}^{* *}(6) Q_{58}^{* *}(7) Q_{81}^{* *}(\mathrm{~s}) Q_{01}^{* *}(\mathrm{~s}) \\
& -Q_{14}^{* *(3)} Q_{45}^{* *}(\mathrm{~s}) Q_{59}^{* *}(\mathrm{~s}) Q_{91}^{* *(10)} Q_{01}^{* *}(\mathrm{~s}) \\
& -Q_{14}^{* *(3)} Q_{45}^{* *}(\mathrm{~s}) Q_{58}^{* *}(7)(\mathrm{s}) Q_{81}^{* *}(\mathrm{~s}) Q_{01}^{* *}(\mathrm{~s}) .
\end{aligned}
$$

And $D_{1}(\mathrm{~s})$ is already specified.

$$
V_{0}=\frac{N_{4}(0)}{D_{1}^{\prime}(0)}=\frac{N_{4}}{D_{1}}
$$

Where,

$$
\begin{aligned}
N_{4}= & 1-p_{12} p_{25}^{(6)} p_{59} p_{91}^{(10)}-p_{12} p_{25}^{(6)} p_{58}^{(7)} \\
& -p_{14}^{(3)} p_{59} p_{91}^{(10)}-p_{14}^{(3)} p_{58}^{(7)} .
\end{aligned}
$$

And $D_{1}$ is defined already.

\section{Cost-benefit Analysis}

The total profit of the system in steady state is given by

$$
\mathrm{P}=C_{0} A_{0}-C_{11} B_{0}^{D}-C_{12} B_{0}^{H}-C_{2} V_{0}
$$

$C_{0}=$ Expected revenue in up time(o,t]

$C_{11}=$ Expected total repair cost when repairman is busy under digging out.

$C_{12}=$ Expected total repair cost when repairman is busy under hospitalization.

$C_{2}=$ Per visit cost of the repairman.

\section{Particular Cases}

Numerical result for the particular cases the following case is considered:

$$
\begin{aligned}
& g_{1}(t)=\alpha_{1} e^{-\alpha_{1} t}, g_{2}(t)=\alpha_{2} e^{-\alpha_{2} t}, \\
& g_{3}(t)=\alpha_{3} e^{-\alpha_{3} t}, \text { and } g_{4}(t)=\alpha_{4} e^{-\alpha_{4} t}
\end{aligned}
$$




$$
\begin{aligned}
p_{01}=1, p_{12} & =\frac{\alpha_{1}}{\lambda_{2}+\alpha_{1}}, p_{14}=\lambda_{2} /\left(\lambda_{2}+\alpha_{1}\right), p_{14}^{(3)}=\frac{\lambda_{2}}{\lambda_{2}+\alpha_{1}} & & p_{25}^{(6)}=\frac{\lambda_{2}}{\lambda_{2}+\alpha_{2}}, p_{45}=1 . p_{59}=\alpha_{3} /\left(\lambda_{1}+\alpha_{3}\right), p_{58}^{(7)}=\frac{\lambda_{1}}{\lambda_{1}+\alpha_{2}}, \\
p_{20} & =\alpha_{2} /\left(\lambda_{2}+\alpha_{2}\right), p_{26}=\lambda_{2} /\left(\lambda_{2}+\alpha_{2}\right), & & p_{81}=1, p_{90}=\alpha_{4} /\left(\lambda_{1}+\alpha_{4}\right), p_{91}^{(10)}=\frac{\lambda_{1}}{\lambda_{1}+\alpha_{4}}
\end{aligned}
$$

\section{Grapical Interpretation}

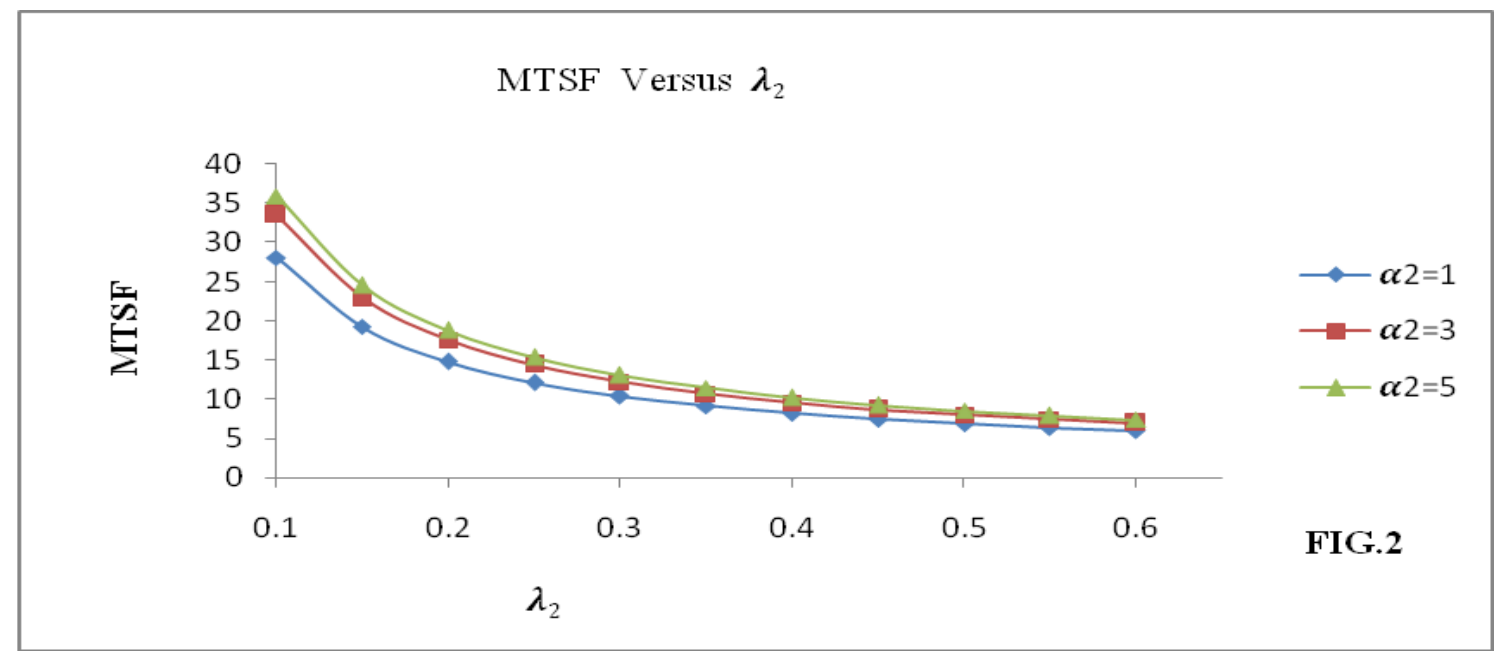

Figure 2.

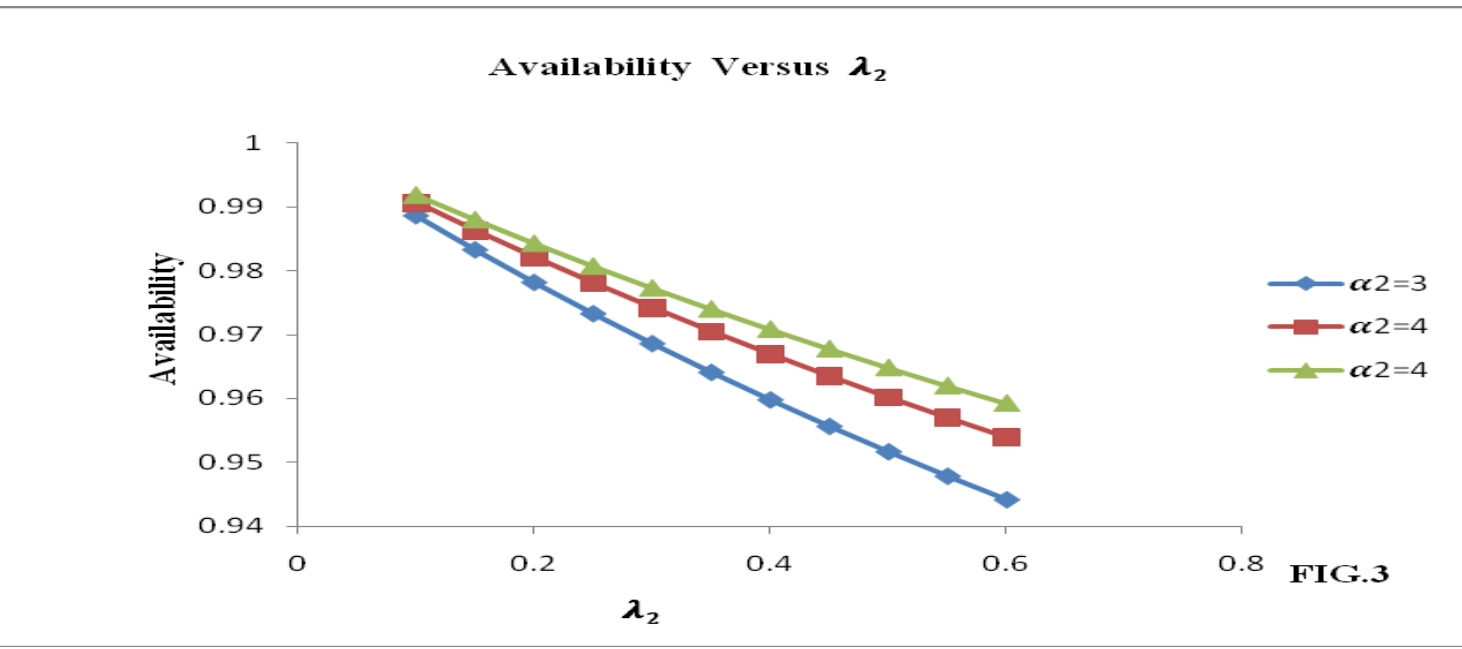

Figure 3.

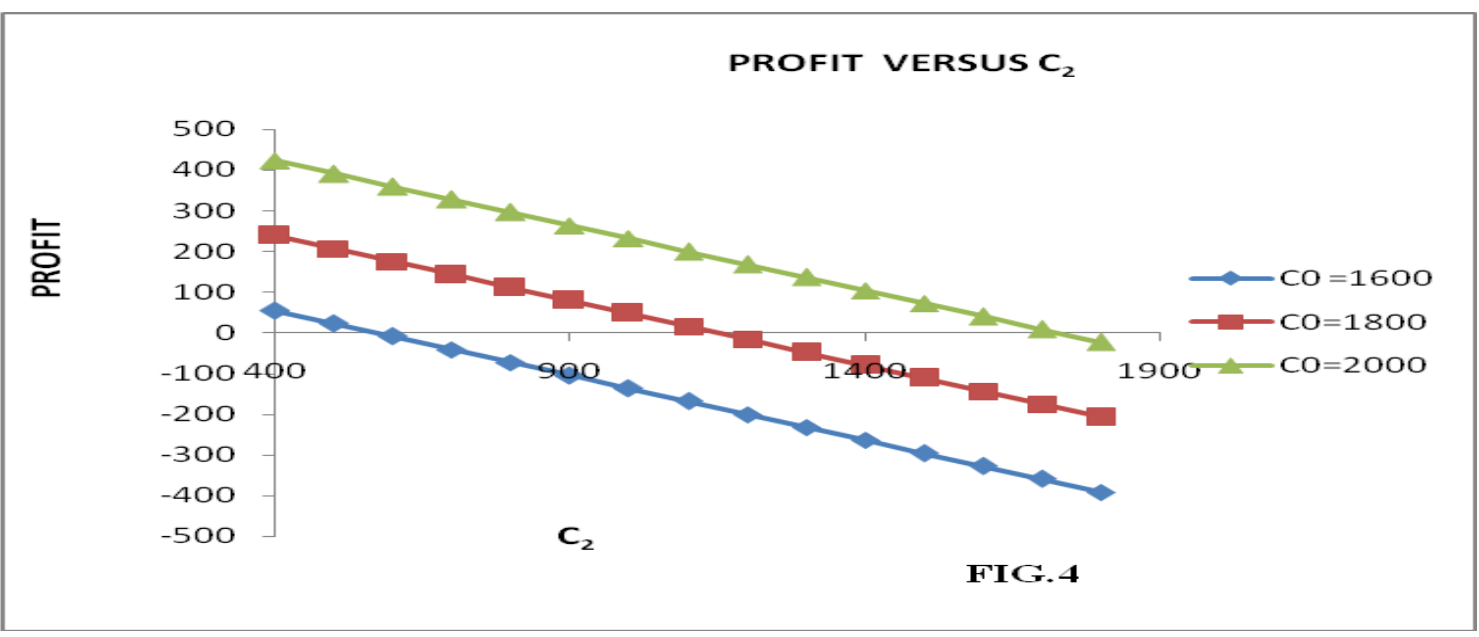

Figure 4 . 


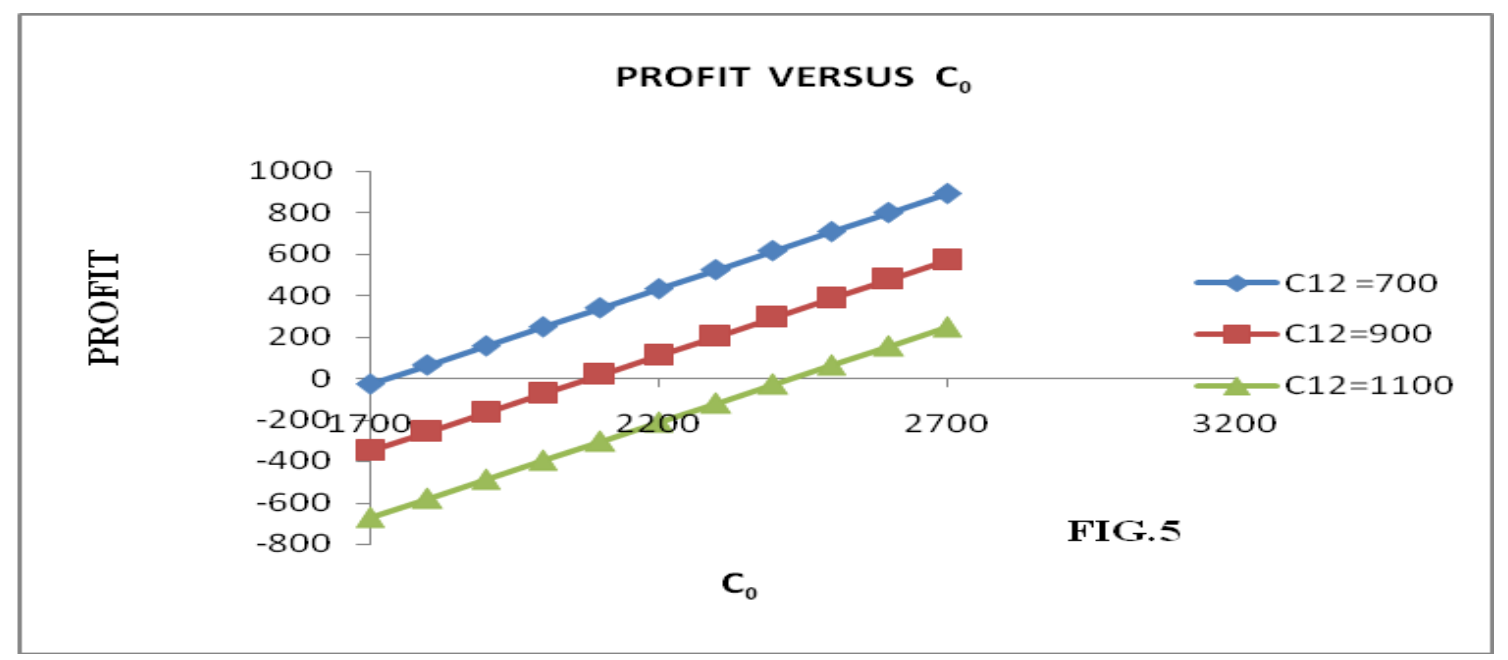

Figure 5.

\section{Conclusion}

For the particular case discussed above when the system is fails due to snow storm the reliability measures of the system such as mean time to system failure, availability, profit are computed. For the particular case discussed above the graphical interpretation are drawn in figures [2-4]. From the Figure 2 and Figure 3 it is observed the the MTSF and availability decreases as the failure rate increases respectively. Also from the Figure 4 profit is decreases as per visit repair rate of the repairman is increases and from the Figure 5 profit is increases as the revenue cost of the per unit is increases.

\section{Acknowledgements}

All authors acknowledge MD university, Rohtak for providing facilities and infrastructure. Further NS acknowledge UGC, New Delhi for their financial support to carry out research work.

\section{References}

[1] Osaki, Reliability Analysis of a Two-Unit Standby-Redundant System with Preventive Maintenance IEEE Transactions on Reliability, 21, 24-29, 1972

[2] Gupta,P.P and Agarwal, S.C A Parllel Redundant Complex System with Two Types of Failure under Preemptive-Repeat Repair Discipline Microelectronics Reliability. 24(3), 395-409, 1984.
[3] Gupta, P.P and Sharma, M.K. Reliability and MTTF Evaluation of a Two Duplex Standby System with Two Types of Repair Microelectronics Reliability, 33(3), 291-305, 1993.

[4] Taneja, G., Tuteja, R.K., and Arora, R.T., Analysis of Two-Unit System with Partial Failures and Three Types of Repair Reliability Engineering and System Safety, 33,199-214,1991.

[5] Taneja, G., Tuteja, R.K., Cost-benefit analysis of a two-server, two-unit, warm standby system with different types of failure Microelectronics Reliability, 32, 1353-1359, 1992.

[6] Singh, D. and Taneja,G. Comparative of a Power Plant comprising one steamturbine with respect to two types of inspection IJSCE, 6, 331-338, 2014.

[7] Malhotra R., Taneja G.,Comparative study between a single unit system and a two - unit cold standby systemwith varying demand, Springer Plus, 4, 1-17, 2015.

[8] Chandrasekhar P., Natrajan R. A study on a two-unit standby system with Erlangian repair time Asia-Pacific Journal of Operational Research, 21(3), 271-277, 2015.

[9] Manocha A. and Taneja G. Stochastic Analysis of a two-unit standby system with arbitrary distributions for life, repair and waiting times 11(3), 293-299, 2015.

[10] Goel L.R., Sharma G.C., Gupta R. Cost analysis of a two-unit cold standby system under different weather conditions Microelectron Reliab, 25(4), 655-659, 1985.

[11] Gupta, R., and Goel, R. Profit analysis of a two-unit cold standby system with abnormal weather condition Microelectronics Reliability, 31(1), 1-5, 1991.

[12] Goel L.R., Kumar A. and Rastogi A. K. Stochastic Behaviour of Man-Machine Systems Operating Under Different Weather Conditions Microelectron Reliab, 25(1), 87-91, 1985.

[13] S.N., S.D and Saini A.K., Cost-Benefit analysis of two identical warm standby system subject to under heavy rain with partially operative after repair International Journal of Pure and Applied Mathematics, 114(3), 503-514, 2017.

[14] Nailwal B. and Singh S.B. Reliability and Sensitivity analysis of an operating system with inspection in different weather conditions International Journal of Reliability and Safety Engineering, 19(2), 1250009 (36 pages), 2014. 